\title{
Tomografía computarizada Cone Beam en endodoncia.
}

\section{Pàmela Oviedo Muñoz ${ }^{1}$ Juan Felipe Hernández Añaños ${ }^{2}$}

Cirujano Dentista. Residente de la Especialidad de Endodoncia. Facultad de Estomatología. Universidad Peruana Cayetano Heredia. Lima, Perú.

${ }^{2}$ Cirujano Dentista. Especialista en Endodoncia. Docente del Postgrado en Endodoncia, Facultad de Estomatología. Universidad Peruana Cayetano Heredia. Lima Perú

\section{Correspondencia}

Pàmela Oviedo Muñoz.

Tarragona 135 Residencial Higuereta -

Lima 33, Perú

Teléfono: 511-975020202

E-mail: pame_om20@hotmail.com

Oviedo-Muñoz $\mathrm{P}^{1}$, Hernández-Añaños $\mathrm{JF}^{2}$. Tomografía computarizada Cone Beam en endodoncia. Rev Estomatol Herediana. 2012; 22(1):59-64.

\section{RESUMEN}

La tomografía computarizada Cone Beam gracias a los avances tecnológicos esta especialmente diseñada para producir imágenes de alta resolución e información tridimensional para aplicaciones dentales, esta tiene ventajas en la detección de los signos clínicos y la precisión en el diagnóstico.

Además reduce el tiempo de exploración y sobre todo reduce la dosis de radiación en comparación de la tomografía computarizada tradicional.

La tomografía computarizada Cone Beam y su aplicación en la endodoncia es muy importante ya que nos proporciona diversos cortes (axial, coronal y sagital) que nos va permitir dar un diagnóstico más preciso sobre fracturas radiculares, reabsorciones radiculares, periodontitis apical, perforaciones durante el tratamiento endodóntico, planificación de cirugías endodónticas y sobre todo nos va ayudar a reconocer la anatomía de las raíces y el número de conductos presentes en cada diente.

Palabras Clave: TOMOGRAFÍA COMPUTARIZADA CONE BEAM (CBCT), APLICACIÓN ENDODÓNTICA.

Cone beam computed tomography in endodontics.

ABSTRACT

Due to technological advances the Cone Beam Computed Tomography (CBCT) has been specially designed to give images in high resolution and tridimensional information. The CBCT has may advantages as it may give high precision images in early diagnosis when compared with traditional computed tomography and conventional radiography. Cone Beam Computed Tomography is very important in endodontic as it gives us different: axial, coronal and sagittal cuts. That will allow us to give a more accurate diagnosis of root fractures, root resorption, apical periodontitis, endodontic perforations during treatment, endodontic surgery planning and above all will help us to recognize the anatomy and number of the root canal present in each tooth.

Keys Words: CONE BEAM COMPUTED TOMOGRAPHY (CBCT), ENDODONTICS APPLICATIONS.

\section{Introducción}

El uso de estudios imaginológicos es importante para el manejo de problemas endodónticos.

Las imágenes proporcionadas por las radiografías convencionales nos brindan información limitada ya que sólo es la representación bidimensional de estructuras tridimensionales. A esto se suma la superposición de estructuras anatómicas adyacentes.

La evolución de la imagen convencional a imágenes $2 \mathrm{D}$ y $3 \mathrm{D}$ otorga beneficios para el paciente y para el clínico. Entre los beneficios para el paciente está el menor tiempo de exposición a la radiación comparando entre la tomografía convencional y la tomografía Cone Beam.

La tomografía Cone Beam aplicado a la endodoncia esta dando muchos beneficios como la exploración previa de la anatomía radicular que permite al especialista tomar mejores decisiones y establecer un adecuado tratamiento. También permite diagnosticar reabsorciones radiculares en las diferentes superficies y tercios radiculares, periodontitis apical en estadios iniciales, fracturas radiculares verticales y horizontales, perforaciones radiculares, identificación de instrumentos separados.

\section{Tomografía computarizada Cone Beam}

Los tomógrafos computarizados Cone Beam (haz cónico) representan el desarrollo tecnológico de un tomógrafo relativamente pequeño y de menor costo, especialmente dedicado para la región dentomaxilofacial. El desarrollo de esta tecnología está permitiendo a los profesionales realizar exploraciones dinámicas de las imágenes adquiridas, incluso la reproducción de una imagen tridimensional de tejidos mineralizados maxilofaciales, con mínima distorsión y dosis de radiación significativamente reducida a comparación de la tomografía tradicional (1).

Se ha demostrado la enorme variación y complejidad del sistema de conductos radiculares, conductos accesorios y múltiples forámenes como hallazgos comunes (2). La interpretación radiológica es esencial en el diagnóstico y plan de tratamiento en endodoncia. La interpretación de una imagen radiográfica puede estar limitada por la anatomía dental interna y las estructuras que lo rodean al ser una imagen bidimensional (3).

La información adquirida en la radiografía convencional y digital se ve limitada ya que la anatomía tridimensional de la zona esta comprimida en una imagen bidimensional. A veces la información esencial de la anatomía tridimensional del diente o dientes y estructuras 
adyacentes es ocultada, incluso con la mejor técnica radiográfica utilizada.

La distorsión y superposición de estructuras dentales en vistas periapicales es inevitable. Las imágenes adquiridas con la tomografía computarizada Cone Beam (CBCT) tienen una mayor sensibilidad que la radiografía convencional, en la evidencia inicial de la enfermedad periapical, al detectar tempranamente estadios iniciales de cambios radiolúcidos versus cambios hipodensos alrededor del ápice radicular.

La CBCT también puede ser utilizada para la planificación de cirugía endodóntica (4). Así también, se puede aplicar para evaluación de la anatomía del sistema de conductos radiculares, evaluación de defecto de reabsorción, evidenciar perforaciones y fracturas radiculares, etc (5). Es importante tener los conocimientos y la habilidad para realizar las exploraciones dinámicas de las imágenes adquiridas con los tomógrafos para evaluar un área de interés. Ello podría resultar en un mayor beneficio tanto a clínicos principiantes como a clínicos experimentados (3).

\section{Presencia de artefactos}

Es un problema significativo, que puede afectar la calidad de imagen y la exactitud diagnóstica de las imágenes $\mathrm{CBCT}$, la dispersión y el endurecimiento del haz, causado por la alta densidad de las estructuras vecinas, tal como el esmalte, postes de metal y restauraciones . Si esta dispersión y endurecimiento del haz se asocia íntimamente con el diente que se evalúa, las imágenes CBCT resultantes pueden tener un valor diagnóstico mínimo (6).
Aplicación de Tomografía Computarizada Cone Beam en el diagnóstico endodóntico.

\section{Anatomía de conductos radiculares}

El principal objetivo del tratamiento de conductos es la preparación mecánica y limpieza química de todo el sistema de conductos radiculares y dentina contaminada, seguida de una adecuada obturación. En la Fig. 1 se muestra una vista axial a nivel del tercio cervical de una primera molar con la obturación de cuatro conductos.

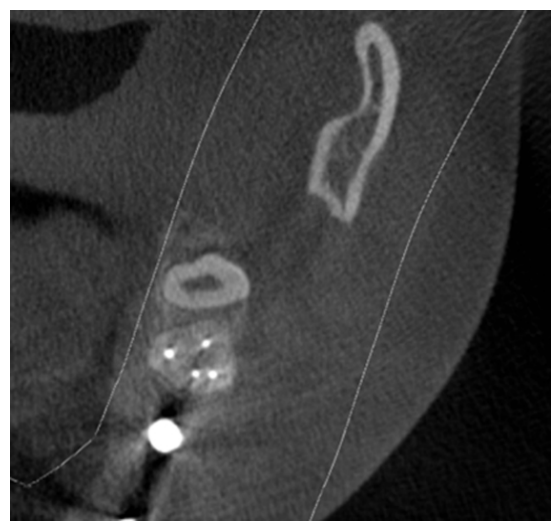

Figura 1. ista axial de la obturación de 4 conductos en una primera molar. png $137 \mathrm{~K}$

Muchas veces durante el trabajo clínico únicamente nos basamos en conceptos teóricos sobre la anatomía dental pero no tomamos en cuenta las variaciones anatómicas que se pueden presentar, lo cual podría conllevar al fracaso del tratamiento. Por eso es importante identificar las variaciones en la anatomía dental antes del tratamiento de conductos. (8)

La et al (2010) reporta un caso donde se identificó el conducto medio mesial de la raíz mesial de una primera molar inferior, la cual tenía tratamiento de conducto y persistencia de sintomatología. Las exploraciones con CBCT de la primera molar permitió obtener la morfología en los cortes transversales, axiales y sagitales de $1.0 \mathrm{~mm}$ de espesor. Las imágenes transversales revelaron que la raíz mesial tenía 3 conductos. (9)

En la Fig.2 se muestra un caso donde se evidencia el conducto medio mesial de la raíz mesial de una molar inferior. Caso obtenido del Postgrado de Endodoncia de la Universidad Peruana Cayetano Heredia.

Kottoor (2010) Reportó un caso en el cual a través de las imágenes de la CBCT identificó 5 orificios de conductos radiculares en una segunda molar superior derecha la cual tenía dos raíces palatinas y 2 raíces mesiobucales separadas y una raíz distobucal. (10)

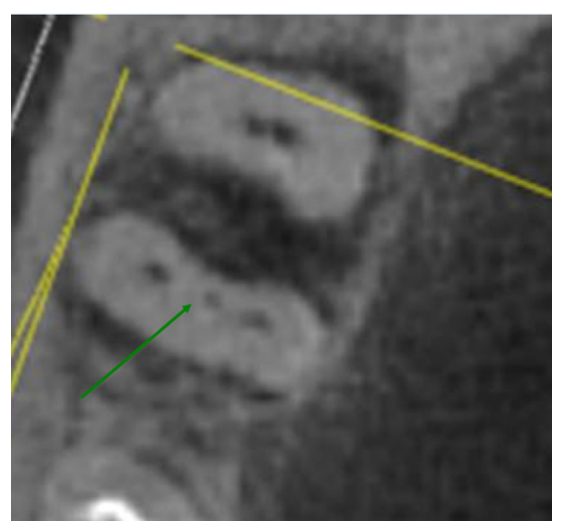

Figura 2. Figura 2- Presencia de conducto medio mesial en una primera molar inferior (Caso del Postgrado de Endodoncia de la Universidad Peruana Cayetano Heredia). jpg $20 \mathrm{~K}$.

\section{Fracturas radiculares}

El diagnóstico de algunas fracturas radiculares en radiografías convencionales puede ser complicado, debido a la falta de signos y síntomas clínicos específicos. Una limitación es la superposición de otras estructuras adyacentes la cual limita la sensibilidad en la detección de fracturas longitudinales, (12). Sin embargo al realizar exploraciones con imágenes de la CBCT se puede observar con nitidez las múltiples 
fracturas que puede presentar el caso. En la Fig. 3a y 3 b se muestra el caso de un paciente en el cual las imágenes tomográficas evidencian múltiples fracturas comparado a la limitación de la radiografía panorámica.

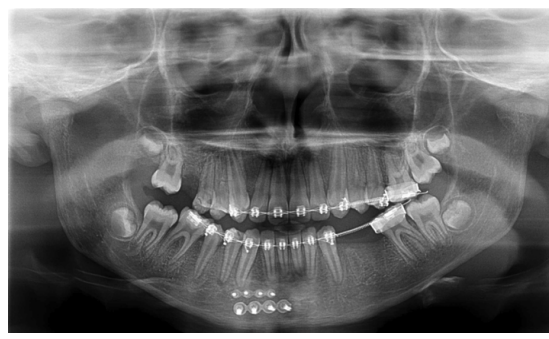

Figura 3a. Radiografía panorámica de un paciente con varios dientes fracturados. No se aprecia con nitidez las fracturas dentarias. (Imagen cortesía del Dr. Luis Raggio). jpg $34 K$

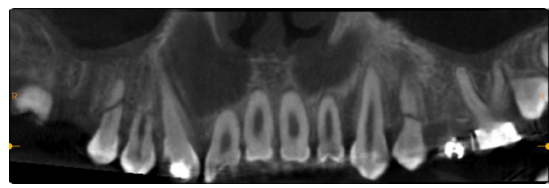

Figura 3b. Tomografía Cone Beam del caso mostrado en la figura $3 a$ en donde se evidencia las fracturas radiculares de varias piezas dentarias. (Imagen cortesía del Dr. Luis Raggio).jpg $18 \mathrm{~K}$

Chen (2008) demostró en un estudio, con 5 años de seguimiento en dientes con tratamiento endodóntico no quirúrgico, donde la fractura radicular fue el hallazgo adverso en un $32.1 \%$ y el tratamiento elegido fue la exodoncia (13).

Hassan (2010) demostró que la sensibilidad global detectada por las exploraciones con CBCT fue más alta en comparación con radiografías periapicales (RP). La especificidad global de las exploraciones con CBCT fue ligeramente menor que RP, pero no significantemente diferente, concluyendo que las exploraciones con CBCT fueron significativamente más precisas que las RP en la detección de fractura vertical radicular (14).

\section{Perforación radicular}

La perforación radicular es la comunicación artificial entre el conducto radicular y los tejidos perirradiculares. Si bien esto puede ocurrir debido a la reabsorción radicular, generalmente es el resultado de daños iatrogénicos realizados durante la preparación de cavidades en la apertura cameral, durante la preparación biomecánica del conducto radicular y durante la preparación post tratamiento para la colocación de poste preformados o colados. La comunicación artificial es un factor potencial para una lesión inflamatoria con la destrucción de los tejidos periodontales adyacentes. El pronóstico del diente con perforación depende principalmente del control de la infección bacteriana en el sitio de la perforación. La evaluación preoperativa, de la presencia de perforación radicular es importante para definir el pronóstico y plan de tratamiento. La detección radiográfica de la superficie vestibular o lingual es dificultosa, porque la imagen de la perforación esta superpuesta en la raíz. Si se toma radiografías pre operativas en distintos ángulos horizontales puede facilitar la identificación en la superficie vestibulo - lingual. (15) Sin embargo el CBCT nos permite dar un diagnóstico mas preciso en este tipo de lesiones. (Fig 4).

Young (2007) reportó el caso de un paciente masculino de 34 años quien presentaba dolor a la percusión vertical en la pieza 21 . La radiografía mostraba que la pza. 21 tenía un poste y una obturación radicular compacta. Entre las posibles etiologías se mencionó la perforación lateral, fractura radicular o filtración de irritantes microbianos a través de un conducto lateral. Para determinar con mayor precisión la relación entre poste y estructura de la raíz, se decidió el uso de imágenes de CBCT. Estas imágenes confirmaron que la pieza 21 tenía una perforación en la superficie vestibular posterior a nivel medio radicular con un defecto óseo asociado. (15)

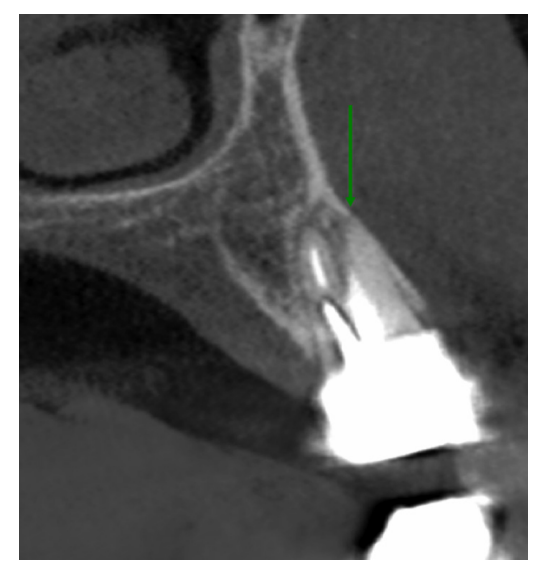

Fig 4. Imagen al corte transversal muestra raíz palatina con obturación de condcuto y poste. La raíz vestibular no obturada. A nivel de furcación presencia de poste. Persistencia de la lesión periapical. $16 K$

\section{Cirugía apical}

El examen clínico, la buena calidad de radiografías periapicales son esenciales para el diagnóstico preoperatorio de los dientes que serán sometidos a cirugía periapical. Sin embargo, la radiografía periapical está limitada, por que sólo es la representación en dos dimensiones. La interpretación es más difícil cuando motivo principal es complejo, esto ocurre a menudo en la región posterior del maxilar, donde la raíces de los dientes se superponen a estructuras anatómicas como el arco cigomático y el seno maxilar. La presencia de materiales de obturaciones radiopacos y postes también se sumaran al problema de fondo (16).

Low (2008), reporta el caso de un paciente masculino de 38 años de edad, quien presenta sintomato- 
logía de la pieza 16 , la radiografía mostró la presencia de un instrumento separado. Se utilizó imágenes de CBCT con las que se evaluó la posición exacta del instrumento fracturado y la relación espacial con el seno maxilar (17).

Humonean et al. (2006) examinaron 39 conductos obturados del maxilar inferior y concluyeron que la CBCT da mas información importante para la decisión de retratamientos, especialmente en los casos para cirugía periapical (18).

Lofthag-Hansen (2007), descubrió lesiones adicionales usando CTCB y reportó que en el 70\% de los casos proporciona información clínica relevante que no fue encontrada en la radiografía periapical (19).

Rigolone et al. concluyeron que CBCT puede jugar un papel importante en la planificación de la microcirugía periapical de las raíces palatinas de los primeros molares superiores. También se podría evaluar y medir la distancia entre la placa cortical y el ápice de la raíz palatina y la presencia o ausencia del seno maxilar entre las raíces (20).

Patel encontró que la proyección de imagen tridimensional permite la relación anatómica de los ápices de las raíces con estructuras anatómicas adyacentes importantes, tales como el conducto del nervio dentario inferior, el agujero mentoniano y el seno maxilar, que se identificaran claramente en cualquier plano que el clínico desea ver (21).

\section{Reabsorción radicular}

La reabsorción radicular es una condición fisiológica o patológica asociada con la perdida de estructura dental causada por las células clásticas. La capa superficial interna o externa de células de protección podrían ser dañadas o sustituidas y la reabsorción radicular podría afectar cualquier parte de la raíz. (Fig. 5 y $5 b$ ) Varios aspectos de la reabsorción radicular inflamatoria, como prevalencia, factores etiológicos, la clasificación basada en la superficie dental, progresión, extensión y los mecanismos patológicos se han discutido ampliamente. Sin embargo la reabsorción radicular inflamatoria es una lesión asintomática que es difícil de diagnosticar y tratar.
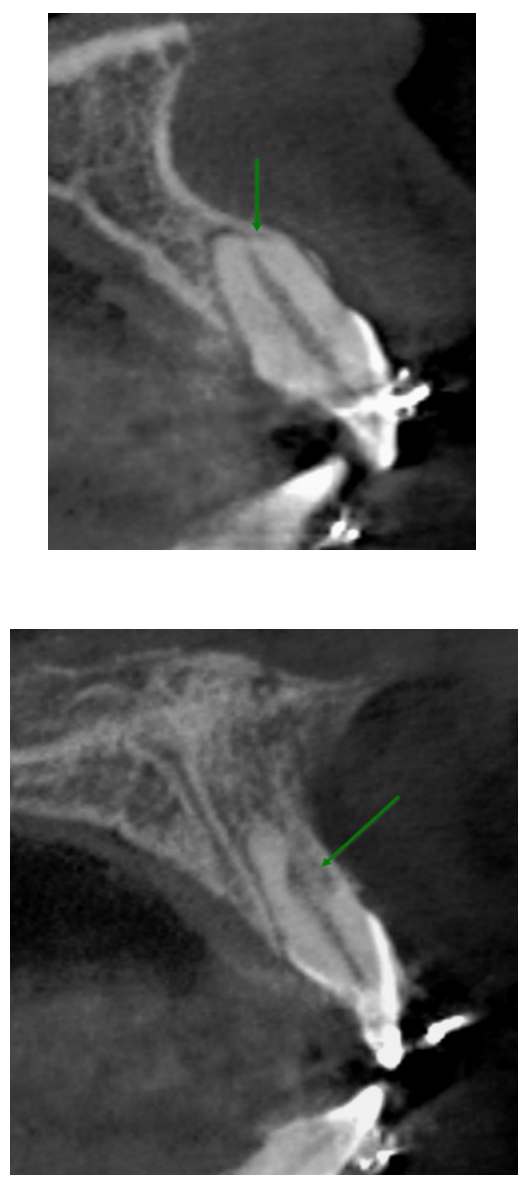

Figura 5. Caso post trauma presenta reabsorción radicular a nivel periapical. Ausencia de tabla ósea vestibular debido a un traumatismo dentario(Fig. 5a) y reabsorción radicular externa (Fig. 5b).jpg $27 \mathrm{~K}$

Frecuentemente se usan radiografías convencionales para diagnosticar y dar tratamiento. Sin embargo el acortamiento apical, ampliación del conducto radicular y las radiolucencias externas de la raíz no son detectables en las radiografías en sus etapas iníciales, cuando son pequeños o por la limitación bidimensional de este método (22)

Estrela (2009) presenta un método para evaluar IRR ( Reabsorcion radicular inflamatoria) usando imágenes de CBCT . El criterio de estudio fue establecido para el analisis del sitio de IRR: Tercio radicular : apical, medio y cervical, superficie radicular : mesial, distal,bucal, palatal o lingual y el ápice radicular y la extensión de IRR. La extension mas grande de RR fue medida y un sistema de puntuación de 5 puntos $(0-4)$ fue usado para el análisis (22).

Nakata (2009) reportó un caso de un paciente masculino de 39 años de edad, quien refiere molestia en la pieza 35 , al examen clínico se evidencia fístula y dolor a la percusión vertical en dicha pieza. La radiografía mostró la presencia de una lesión periradicular con leve reabsorción ósea en la cara distal del premolar. Se obtuvieron imagen con la CBCT, donde se observo la reabsorción radicular externa en las superficies bucal, mesial y distal de la raíz (23)

Salatino (2009) evaluó la influencia del tamaño del voxel en la detección de reabsorciones radiculares probando así la capacidad de diagnóstico de CBCT para detectar reabsorciones radiculares externas. Los resultados obtenidos indican que los valores de sensibilidad y especificidad de CBCT fueron similares y no dependen de la resolución de voxel (24). 


\section{Periodontitis apical}

La radiografía periapical es un recurso esencial en el diagnostico endodóntico, ya que ofrece evidencia importante de la progresión, regresión y persistencia de periodontitis apical (PA) (25).

Algunos investigadores demostraron que un quiste puede distinguirse de un granuloma periapical con $\mathrm{CBCT}$ porque nos muestra una marcada diferencia en densidad entre el contenido de la cavidad del quiste y el tejido granulomatoso, favoreciendo así un diagnostico no invasivo (26).

Orstavik (1986) presenta un índice periapical (PAI) como un sistema de puntuación para la evaluación radiográfica de periodontitis apical. . El PAI representa una escala ordinal de 5 puntuaciones que van desde ausencia de enfermedad hasta periodontitis severa con características exacerbadas y están basadas en referencias radiográficas y confirmadas por diagnóstico histológico. Dada las limitaciones de la radiología convencional para la detección de AP y la disponibilidad de nuevas modalidades de imágenes tridimensionales, parece ser una necesidad el desarrollo de un nuevo índice periapical (27).

Estrela (2008) propone en un estudio un índice periapical para imágenes de Tomografía Computarizada Cone Beam (CBCTPAI), que fue desarrollado en base a los criterios establecidos de mediciones correspondientes a imágenes radiolúcidas periapicales interpretados en CBCT. El tamaño de la imagen radiolúcida sugiere una lesión periapical que fue delimitada y medida, usando un sistema de puntuación de 6 puntos $(0-5)$. Además, teniendo en cuenta que la CBCT proporciona imágenes en 3 dimensiones, se incluyó dos variables como expansión de la cortical del hueso (E) y destrucción de la cortical del hueso (D).

Estrela (2008) evaluó la prevalencia de periodontitis apical en imágenes de radiografías convencionales y cortes de CBCT. Teniendo como resultado que la prevalencia de periodontitis apical identificado por la radiografía periapical y por CBCT usando los criterios de CBCTPAI fue de $39.5 \%$ y $60.9 \%$ respectivamente (28).

Estrela (2008) evaluó la precisión de la tomografía computarizada cone beam y las radiografías panorámica y periapicales para la detección de la periodontitis apical, teniendo como resultado que la tomografía computarizada Cone Beam presenta mayor precisión $(63,3 \%)$ en la detección de periodontitis apical en compraración con la Rx. Panorámica $(17,6 \%)$ y Rx. Periapical $(35,3 \%)(29)$.

Wanderley (2009) evaluó la radiográfica periapical y CBCT en el diagnóstico de periodontitis apical usando hallazgos histopatológicos como un gold estándar. Los resultados obtenidos fueron que la radiografía periapical diagnosticó el $71 \%$, mientras que las exploraciones con CBCT fueron el $84 \%$ e histológicamente el 93\%. Concluyendo que la tomografía Cone Beam es un buen método para el diagnóstico de periodontitis apical (30).

\section{Conclusiones}

* La tomografía computarizada Cone Beam está diseñada para producir información útil al odontólogo, teniendo ventajas sobre la TC convencional y las radiografías.

* El uso de la tomografía computarizada Cone Beam en odonto- logía es importante, pues reemplaza las imágenes $2 \mathrm{D}$ de las radiografías por una secuencia de imágenes $2 \mathrm{D}$ y $3 \mathrm{D}$, siendo estas de alta resolución para un buen diagnóstico.

* La imágenes con tomografía computarizada Cone Beam permiten ver patologías, que en la radiografía convencional no siempre se puede evidenciar, como son las lesiones de periodontitis apical desde estadios iníciales.

* Las exploraciones con tomografía computarizada Cone Beam muestran alta eficacia en la identificación de la anatomía radicular, reabsorción radicular, fractura radicular, perforación radicular y otros.

\section{Referncias Bibliograficas}

1. Gamba D, Raymundo R, Vasconcellos M, Vasconcellos D, Niza S. Tomografía computadorizada de feixe cônico (Cone beam): entendendo este novo método de diagnóstico por imagem com promisora aplicabilidade na Ortodontia. Rev. Dent. Ortodon Ortop Fac. 2007; 12: 139-156.

2. Kau C, Richmond S. Three-dimensional cone beam computerized tomography in orthodontics. J. Orth. 2005; 32; 4: 282-93.

3. Schramm A. The use of cone beam CT in cranio-maxillofacial surgery. Int. Congress Series. 2005; 4: 1200-1204

4. Rhodes J, Ford T, Lynch J, Liepins P, Curtis R. Micro-computed tomography: a new tool for experimental endodontology. Int. End. J. 1999;32: 165 -70.

5. Cotton TP, Geisler TM, Holden DT, et al. Endodontic application of cone-bean volumetric tomography. J Endod 2007; 33: 112132.

6. Gamba D, Raymundo R, Vascon- 
cellos M, Vasconcellos D, Niza S. Tomografía computadorizada de feixe cônico (Cone beam): entendendo este novo método de diagnóstico por imagem com promisora aplicabilidade na Ortodontia. Rev Dent Ortodon Ortop Fac. 2007; 12: 139-156.

7. Patel S, Dawood A, Whaites E. The potential applications of cone beam computed tomography in the management of endodontic problems. Int End. J. 2007 ; 40: 818-30.

8. Patel S, Dawood A, Whaites E. New dimensions in endodontic imaging: Part 1 Conventional and alternative radiographic systems. Int End J. 2009;42:447- 462.

9. La S, Jung D, Kim E, Min K. Identification of independent middle mesial canal in mandibular first molar using cone-beam computed tomography Imaging. J Endod. 2010;36(3):542-5.

10. Kottoo J, Hemamalathi S, Sudha R, Velmurugan N. Maxillary second molar with 5 roots and 5 canals evaluated using cone beam computerized tomography: a case report. Oral Surg Oral Med Oral Pathol Oral Radiol Endod 2010; 109: 162-5.

11. Estrela $C$, Bueno $M$, Damião $M$, Sousa M, Pécora J. Method for Determination of Root Curvature Radius Using Cone-Beam Computed Tomography Images. Braz Dent J. 2008; 19: 114-18.

12. Bernardes R. Use of cone - beam volumtric tomography in the diagnosis of root fractures. Oral Surg Oral Med Oral Pathol Oral Radiol Endod. 2009;108:270-77.

13. Chen SC, Chueh LH, Hsiao CK, et al. First untoward events and reasons for tooth extraction after nonsurgical endodontic treatment in Taiwan. J Endod. 2008;34:6714.

14. Hassan B. Comparison of Five Cone Beam Computed Tomography Systems for the Detection of
Vertical Root Fractures . J Endod. 2010;36:126-29.

15. Young G. Contemporary management of lateral root perforation diagnosed with the aid of dental computed tomography. Aust Endod J 2007; 33: 112-18.

16. Tsurumachi T, Honda K. A new cone beam tomography system for use in endodontic surgery. Int End J. 2007; 40: 224-32.

17. Low K, Dula K, Burguin W, Arx T. Comparison of periapical radiography and limited cone-beam tomography in posterior maxillary teeth referred for apical surgery. J Endod. 2008;34: 557- 62.

18. Huumonen S, Grondahl K, Molander A. Diagnostic value of computed tomography in retreatment of root fillings in maxillary molars. Int Endod J. 2006;39:827-33.

19. Lofthag-Hansen S, Huumonen S, Grondahl K, Grondahl H. Limited cone-beam CT and intraoral radiography for the diagnosis of periapical pathology. Oral Surg Oral Med Oral Pathol Oral Radiol Endod. 2007;103:114-9.

20. Rigolone M, Pasqualini D, Bianchi L, Berutti E, Bianchi S. Vestibular surgical access to the palatine root of the superior first molar: "low-does cone-beam" CT analysis of the pathway and its anatomic variations. J Endod. 2003;29: 773-5.

21.Patel S, Dawood A, Whaites E, Pitt Ford T. The potential applications of cone beam computed tomography in the management of endodontic problems. International Endodontic Journal. 2007; 40, 818-30.

22. Estrela C. Method to evaluate inflammatory root resorption by using cone beam computed tomography. J Endod. 2009; 35: 14917.

23. Nakata $K$, Naitob $M$, Izumi $M$, Ariji E, Nakamura H. Evaluation of correspondance of dental computed tomography imaging to anatomic observation of external rootreabsortion. J. Endod. 2009; 35:1594-97.

24. Salatino G. Influence of Voxel size in the diagnostic ability of cone beam tomography to evaluate simulated external root resorption. J. Endod. 2009; 35:233- 35.

25. Sampaio M, Aguirre O, Goncalves A, Correa B, Estrela C. Influence of Length of Root Canal Obturation on Apical Periodontitis Detected by Periapical Radiography and Cone Beam Computed Tomography. J Endod. 2009; 35:805-09.

26. Laux M, Abbott PV, Pajarola G, Nair P. Apical inflammatory root resorption: a correlative radiographic and histological assessment. Int Endod J. 2000;33: 483-93.

27. Orstavik D, Kerekes K. The periapical index: a scoring system for radiographic assessment of apical periodontitis. Endod Dent Traumatol. 1986; 2(1):20-34.

28. Estrela C, Reis B, Correa B, Ribamar J, Djalma J. A new periapical index based on cone beam computed tomography. J Endod 2008; 34(11):1325-31.

29. Estrela C, Reis B, Rodrigues C, Azevedo B, Ribamar J. Accuracy of cone beam computed tomography, panoramic and periapical radiographic for the detection of apical periodontitis. J Endod. 2008; 34:273-9.

30. Wanderley F. Accuracy of periapical radiography and cone-beam computed tomography scans in diagnosing apical periodontitis Using histopathological findings as a gold standard. J.Endod. 2009; 35:1009 - 12 . 